\title{
A D-N Alternating Algorithm for Exterior Laplace Problem with Mixed Boundary Value Conditions
}

\author{
Quan Zheng and Huihui Jia* \\ College of Sciences, North China University of Technology, Beijing 100144, China \\ ${ }^{*}$ Corresponding author
}

\begin{abstract}
This paper develops a Dirichlet-Neumann (D-N) alternating algorithm to solve the mixed boundary value problem of Laplace equation in an infinite domain, and analyses the convergence of the algorithm. By choosing a circle surrounding the original boundary as an artificial boundary to divide the unbounded domain into two sub-domains, we can make use of the natural boundary reduction (NBR) method in the infinite subdomain to solve a Dirichlet boundary value problem while use the finite element method in the finite sub-domain to solve a mixed boundary value problem. We prove that the algorithm is convergent geometrically for any relaxation factor between 0 and 1. The numerical experiment results also display that the sequence of iterative solutions is geometrically convergent, the convergence rate is independent of the finite element mesh size $h$, and the maximum nodal error on $\bar{\Omega}_{1}$ is roughly of $O\left(h^{2}\right)$.
\end{abstract}

Keywords-exterior Laplace problem; mixed boundary value conditions; D-N alternating algorithm; natural boundary reduction; Convergence analysis

\section{INTRODUCTION}

Many applications in science and engineering fields, such as hydrodynamics, electromagnetism, acoustics, etc., can be reduced to exterior boundary value problems of partial differential equations. It's very effective to solve boundary value problems on bounded domains by the finite element method and the finite difference method, yet those methods are difficult to be applied to problems on unbounded domains directly. Therefore, a variety of numerical methods are proposed to solve the exterior problems $[1,2,3]$. Among them, the D-N alternating algorithm has been studied to solve problems on unbounded domains, for examples, Laplace equation, Poisson equation and anisotropic elliptic equation with Dirichlet boundary condition $[4,5,6]$, Helmholtz equation with Neumann boundary condition [7,8], and the anisotropic problem with mixed boundary condition in an infinite domain with a concave angle [9].

In this paper, we consider the exterior Laplace problem with mixed boundary value conditions:

$$
\begin{cases}\frac{\partial^{2} u}{\partial x^{2}}+\frac{\partial^{2} u}{\partial y^{2}}=0, & \text { in } \Omega, \\ u=u_{0}, & \text { on } \Gamma_{D}, \\ n_{x} \frac{\partial u}{\partial x}+n_{y} \frac{\partial u}{\partial y}=g, & \text { on } \Gamma_{N}, \\ \lim _{\sqrt{x^{2}+y^{2}} \rightarrow \infty} u \text { is limited, }\end{cases}
$$

where $\Gamma_{D} \cup \Gamma_{N}=\Gamma_{0}, \Gamma_{0}$ is the planar piecewise smooth closed curve, $u_{0} \in H^{\frac{1}{2}}\left(\Gamma_{D}\right)$ and $g \in H^{-\frac{1}{2}}\left(\Gamma_{N}\right)$ are given functions. $\Omega$ is the unbounded domain outside $\Gamma_{0}$. $n=\left(n_{x}, n_{y}\right)$ is the outward unit normal vector on $\Gamma_{0}$. Let meas $\Gamma_{D} \neq 0$ and the function $u$ is bounded at infinity, then the problem (1) has unique solution.

Section 2 designs the D-N alternating algorithm; Section 3 analyzes the convergence of the D-N alternating algorithm; Section 4 displays some numerical results to check the accuracy and effectiveness of this algorithm.

\section{The D-N Alternating AlgORIthm BASED ON NBR}

For solving the problem (1), we make the artificial boundary to be a circle $\Gamma_{1}=\left\{(x, y) \mid x^{2}+y^{2}=R^{2}\right\}$ to enclose $\Gamma_{0}$ and $\operatorname{dist}\left(\Gamma_{0}, \Gamma_{1}\right)>0$, divide $\Omega$ into two parts: inner subdomain $\Omega_{1}$ and outer subdomain $\Omega_{2}$, and then propose the following D-N alternating algorithm:

Step 1. For any initial value $\lambda^{0} \in H^{\frac{1}{2}}\left(\Gamma_{1}\right)$, put $k=0$.

Step 2. Solve a Dirichlet boundary value problem in domain $\Omega_{2}$ : 


$$
\begin{cases}\frac{\partial^{2} u_{2}^{(k)}}{\partial x^{2}}+\frac{\partial^{2} u_{2}^{(k)}}{\partial y^{2}}=0, & \text { in } \Omega_{2}, \\ u_{2}^{(k)}=\lambda^{(k)}, & \text { on } \Gamma_{1} .\end{cases}
$$

Step 3. Solve a mixed boundary value problem in domain $\Omega_{1}$ :

$$
\begin{cases}\frac{\partial^{2} u_{1}^{(k)}}{\partial x^{2}}+\frac{\partial^{2} u_{1}^{(k)}}{\partial y^{2}}=0, & \text { in } \Omega_{1}, \\ \frac{\partial u_{1}^{(k)}}{\partial n_{1}}=-\frac{\partial u_{2}^{(k)}}{\partial n_{2}}, & \text { on } \Gamma_{1}, \\ \frac{\partial u_{1}^{(k)}}{\partial n}=g, & \text { on } \Gamma_{N}, \\ u_{1}^{(k)}=u_{0}, & \text { on } \Gamma_{D} .\end{cases}
$$

Step 4. Input a relaxation factor $\theta_{k}$ and set

$$
\lambda^{(k+1)}=\left.\theta_{k} u_{1}^{(k)}\right|_{\Gamma_{1}}+\left(1-\theta_{k}\right) \lambda^{(k)}, \quad 0 \leq \theta_{k} \leq 1 .
$$

Step 5. Put $k=k+1$, go to Step 2 .

Noting that, only the normal derivative on boundary $\Gamma_{1}$ of the solution of (2) is needed for solving (3). So, instead of solving problem (2), we use the natural integral equation in [2] to obtain $\frac{\partial u_{2}^{(k)}}{\partial n}$ directly by using $\lambda^{k}$ on boundary $\Gamma_{1}$ :

$$
\frac{\partial u_{2}^{(k)}}{\partial n}=-\kappa_{2}\left(\lambda^{(k)}\right)
$$

where

$$
\kappa_{2}\left(\lambda^{(k)}\right)=\frac{1}{4 \pi R} \int_{0}^{2 \pi} \frac{\lambda^{(k)}\left(\varphi^{\prime}\right)}{\sin ^{2} \frac{\varphi-\varphi^{\prime}}{2}} d \varphi^{\prime}
$$

III. THE DiSCRETE VARIATIONAL FORM AND ITS CONVERGENCE

Discrete variational form of the problem (2) and (3) is: find $u_{1, h}^{(k)} \in V_{h}\left(\Omega_{1}\right)$, such that $\forall v_{h} \in V_{1, h}\left(\Omega_{1}\right)$

$$
D_{1}\left(u_{1, h}^{(k)}, v_{h}\right)=\int_{\Gamma_{N}} g v_{h} d s-\int_{\Gamma_{1}} v_{h} \kappa_{2}\left(\lambda^{(k)}\right) d s,
$$

where

$$
\begin{aligned}
& D_{1}\left(u_{1, h}^{(k)}, v\right)=\int_{\Omega_{1}} \nabla u_{1, h}^{(k)} \cdot \nabla v d x d y, \\
& \left.\lambda_{h}^{(k)}\right|_{\Gamma_{1}}=\theta_{k-1} u_{1, h}^{(k-1)}+\left(1-\theta_{k-1}\right) \lambda_{h}^{(k-1)}(k=1,2, \cdots), \\
& V_{h}\left(\Omega_{1}\right)=\left\{v_{h}\left|v_{h} \in H^{1}\left(\Omega_{1}\right), v_{h}\right|_{\Gamma_{D}}=u_{0}\right\}, \\
& V_{1, h}\left(\Omega_{1}\right)=\left\{v_{h}\left|v_{h} \in H^{1}\left(\Omega_{1}\right), v_{h}\right|_{\Gamma_{D}}=0,\left.v_{h}\right|_{\Gamma_{1}}=0\right\} .
\end{aligned}
$$
follows:

From the problem (6), we can get algebraic equations as

$$
\left(\begin{array}{ccc}
K_{11} & K_{1 i} & 0 \\
K_{i 1} & K_{i i} & K_{i 0} \\
0 & K_{0 i} & K_{00}
\end{array}\right)\left(\begin{array}{c}
U_{1, h}^{(k)} \\
U_{i, h}^{(k)} \\
U_{0, h}^{(k)}
\end{array}\right)=\left(\begin{array}{c}
-K_{h} \Lambda^{(k)} \\
0 \\
F_{0}
\end{array}\right),
$$

and

$$
\Lambda^{(k+1)}=\theta_{k} U_{1}^{(k)}+\left(1-\theta_{k}\right) \Lambda^{(k)}, \quad(k=0,1, \cdots),
$$

where the third-order block matrix on the left is a stiffness matrix obtained by finite element method in $\Omega_{1}, K_{h}$ on the right is gotten from the natural boundary element method on $\Gamma_{1}, \Lambda^{(k)}$ is a vector which is constructed by the nodal values of $\lambda^{(k)}$ on $\Gamma_{1}, F_{0}$ is the total load vector on $\Gamma_{N} \cdot U_{1, h}^{(k)}$, $U_{i, h}^{(k)}$ and $U_{0, h}^{(k)}$ are the functional vector to be solved on $\Gamma_{1}$, in $\Omega_{1}$ and on $\Gamma_{N}$ respectively.

Theorem 3.1 The discrete D-N alternating algorithm (7) and (8) is equivalent to the preprocessing Richardson iteration method:

$$
S_{1, h}\left(\Lambda^{(k+1)}-\Lambda^{(k)}\right)=\theta_{k}\left(\bar{F}_{0}-S_{h} \Lambda^{(k)}\right)
$$

where

$$
\begin{aligned}
& S_{1, h}=K_{11}-K_{1 i}\left(K_{i i}-K_{i 0} K_{00}^{-1} K_{0 i}\right)^{-1} K_{i 1}, \\
& S_{h}=S_{1, h}+K_{h}, \\
& \bar{F}_{0}=K_{1 i}\left(K_{i i}-K_{i 0} K_{00}^{-1} K_{0 i}\right)^{-1} K_{i 0} K_{00}^{-1} F_{0} .
\end{aligned}
$$

Theorem 3.2 The condition number of the iterative matrix $S_{1, h}^{-1} S_{h}$ of the discrete D-N alternating algorithm is 
independent of the finite element mesh size $h$.

Theorem 3.3 If $0<\min \theta_{k} \leq \max \theta_{k}<1$, then the discrete D-N alternating algorithm (7) and (8) is convergent, and the convergence rate is independent of the finite element mesh size $h$.

\section{NumERICAL RESUltS}

Example. Consider to solve the problem (1), where $\Omega$ is the infinite domain outside of square $[-1,1] \times[-1,1]$, $\Gamma_{N}=\{(x, y) \mid x=-1,-1 \leq y \leq 1\} \quad$ and $\quad \Gamma_{D}=\Gamma_{0} \backslash \Gamma_{N}$. The exact solution is $u=\frac{x}{x^{2}+y^{2}}$, and consequently $g$ and $u_{0}$ are determined by $u$.

Choosing the artificial boundary as $\Gamma_{1}=\{(r, \theta) \mid r=R$, $R>\sqrt{2}\}$, and making uniform partitions on $\Gamma_{0}, \Gamma_{1}$ and $\Omega$, we have four meshes: $h: 8 \times 2 ; h / 2: 16 \times 4 ; h / 4: 32 \times 8$; $h / 8: 64 \times 16$, and then use the discrete D-N alternating algorithm (7) and (8).

$e_{h}(k)$ denotes the maximum nodal error on $\bar{\Omega}_{1}$ :

$$
e_{h}(k)=\sup _{P_{i} \in \bar{\Omega}_{1}}\left|u\left(P_{i}\right)-u_{1, h}^{(k)}\left(P_{i}\right)\right|
$$

$d_{h}(k)$ denotes the maximum nodal error of the adjacent twosteps:

$$
d_{h}(k)=\sup _{P_{i} \in \bar{\Omega}_{1}}\left|u_{1, h}^{(k)}\left(P_{i}\right)-u_{1, h}^{(k-1)}\left(P_{i}\right)\right|
$$

$q_{h}(k)$ denotes the approximation of the convergence rate:

$$
q_{h}(k)=\frac{d_{h}(k-1)}{d_{h}(k)} .
$$

The numerical results are as follows (Table 1, Table 2 and Table 3):

TABLE I. THE RATIOS OF ERRORS BETWEEN $u_{1, h}^{(k)}$ AND $u$ W.R.T. $h$

\begin{tabular}{ccccc}
\hline Ratio & \multicolumn{4}{c}{$k$} \\
\cline { 2 - 5 } & 2 & 3 & 4 & 5 \\
\hline$e_{h}(k) / e_{h / 2}(k)$ & 1.72693 & 1.70784 & 1.70310 & 1.70189 \\
\hline$e_{h / 2}(k) / e_{h / 4}(k)$ & 2.84887 & 2.72295 & 2.69462 & 2.68784 \\
\hline$e_{h / 4}(k) / e_{h / 8}(k)$ & 3.62506 & 3.55190 & 3.41010 & 3.37851 \\
\hline
\end{tabular}

TABLE II. THE RELATIONSHIP BETWEEN MESH SIZE AND CONVERGENCE RATE $(\mathrm{R}=4, \Theta=0.4)$

\begin{tabular}{ccccrrrrrr}
\hline mesh & $k$ & & 0 & 1 & 2 & 3 & 4 & 5 & 6 \\
\hline$h$ & $e_{h}(k)$ & 0.33029 & 0.25084 & 0.25705 & 0.25873 & 0.25919 & 0.25931 & 0.25935 & 0.25936 \\
& $d_{h}(k)$ & & 0.18062 & 0.05057 & 0.01417 & 0.00397 & 0.00112 & 0.00034 & 0.00010 \\
& $q_{h}(k)$ & & & 3.57187 & 3.56970 & 3.56604 & 3.56097 & 3.29301 & 3.24789 \\
\hline$h / 2$ & $e_{h}(k)$ & 0.23615 & 0.13866 & 0.14885 & 0.15150 & 0.15219 & 0.15237 & 0.15242 & 0.15243 \\
& $d_{h}(k)$ & & 0.16664 & 0.04237 & 0.01096 & 0.00289 & 0.00076 & 0.00020 & 0.00005 \\
& $q_{h}(k)$ & & & 3.93341 & 3.86506 & 3.78821 & 3.78758 & 3.78615 & 3.78412 \\
\hline$h / 4$ & $e_{h}(k)$ & 0.22805 & 0.05928 & 0.05225 & 0.05564 & 0.05648 & 0.05669 & 0.05674 & 0.05675 \\
& $d_{h}(k)$ & & 0.17702 & 0.04258 & 0.01032 & 0.00253 & 0.00064 & 0.00016 & 0.00004 \\
& $q_{h}(k)$ & & & 4.15739 & 4.12677 & 4.07897 & 3.95567 & 3.95830 & 3.96027 \\
\hline$h / 8$ & $e_{h}(k)$ & 0.24045 & 0.05468 & 0.01441 & 0.01566 & 0.01656 & 0.01678 & 0.01683 & 0.01684 \\
& $d_{h}(k)$ & & 0.18578 & 0.04315 & 0.01011 & 0.00238 & 0.00058 & 0.00014 & 0.00004 \\
& $q_{h}(k)$ & & & 4.30508 & 4.27046 & 4.23996 & 4.09522 & 4.06111 & 4.06448 \\
\hline
\end{tabular}


TABLE III. THE RELATIONSHIP BETWEEN RELAXATION FACTOR $\Theta$ AND CONVERGENCE RATE ( $\mathrm{R}=4, \mathrm{~N}=16, \mathrm{P}=4)$

\begin{tabular}{|c|c|c|c|c|c|c|c|c|c|}
\hline$\theta$ & $k$ & 0 & 1 & 2 & 3 & 4 & 5 & 6 & 7 \\
\hline \multirow[t]{3}{*}{0.45} & $e_{h}(k)$ & 0.23615 & 0.14358 & 0.15094 & 0.15218 & 0.15239 & 0.15243 & 0.15243 & 0.15243 \\
\hline & $d_{h}(k)$ & & 0.18747 & 0.03044 & 0.00523 & 0.00090 & 0.00016 & 0.00003 & 0.00001 \\
\hline & $q_{h}(k)$ & & & 6.15826 & 5.81721 & 5.81448 & 5.80546 & 5.79278 & 5.77763 \\
\hline \multirow[t]{3}{*}{0.5} & $e_{h}(k)$ & 0.23615 & 0.14850 & 0.15212 & 0.15241 & 0.15243 & 0.15243 & 0.15243 & 0.15243 \\
\hline & $d_{h}(k)$ & & 0.20830 & 0.01575 & 0.00125 & 0.00010 & 0.00001 & 0.00000 & 0.00000 \\
\hline & $q_{h}(k)$ & & & 13.22463 & 12.57553 & 12.35673 & 11.82296 & 10.41002 & 8.95196 \\
\hline \multirow[t]{3}{*}{0.55} & $e_{h}(k)$ & 0.23615 & 0.15342 & 0.15239 & 0.15244 & 0.15243 & 0.15243 & 0.15243 & 0.15243 \\
\hline & $d_{h}(k)$ & & 0.22913 & 0.00583 & 0.00029 & 0.00002 & 0.00000 & 0.00000 & 0.00000 \\
\hline & $q_{h}(k)$ & & & 39.32322 & 20.27816 & 14.78793 & 12.86079 & 13.97590 & 10.39517 \\
\hline \multirow[t]{3}{*}{0.6} & $e_{h}(k)$ & 0.23615 & 0.15834 & 0.15175 & 0.15252 & 0.15242 & 0.15243 & 0.15243 & 0.15243 \\
\hline & $d_{h}(k)$ & & 0.24996 & 0.02966 & 0.00370 & 0.00049 & 0.00007 & 0.00001 & 0.00000 \\
\hline & $q_{h}(k)$ & & & 8.42807 & 8.01647 & 7.56591 & 7.11229 & 6.71366 & 6.38820 \\
\hline \multirow[t]{3}{*}{0.65} & $e_{h}(k)$ & 0.23615 & 0.16326 & 0.15019 & 0.15290 & 0.15233 & 0.15246 & 0.15243 & 0.15243 \\
\hline & $d_{h}(k)$ & & 0.27079 & 0.05737 & 0.01239 & 0.00273 & 0.00062 & 0.00014 & 0.00003 \\
\hline & $q_{h}(k)$ & & & 4.71983 & 4.63229 & 4.53461 & 4.42881 & 4.31954 & 4.21179 \\
\hline
\end{tabular}

From the numerical results, we can see that:

(1) Table 1 shows that the sequence of iterative solutions is geometrically convergent according to the decrease rate $q_{h}(k)$ of $d_{h}(k)$; moreover, when using different mesh size, the convergence speed with the same relaxation factor is approximately same, which is consistent with Theorem 3.2.

(2) Table 2 shows the finer the mesh size is, the smaller the error between the iterative convergence solution and the accurate solution will be, and the maximum nodal error on $\bar{\Omega}_{1}$ is roughly of $O\left(h^{2}\right)$;

(3) Table 3 shows that when $\theta$ closes to 0.55 , the convergence rate of the iterative algorithm will be faster.

\section{ACKNOWLEDGMENT}

The author would like to thank the referees for their helpful suggestions. This paper is supported by Natural Science Foundation of China (No. 11471019).

\section{REFERENCES}

[1] A. Bayliss, M. Gunzburger, E. Turkel, "Boundary conditions for the numerical solution of elliptic equations in exterior regions", SIAMJ. Appl. Math., vol. 42, no. 2, pp. 430-451, 1982.

[2] H. D. Han, X. N. Wu, Artificial Boundary Method, Tsinghua Univ. Pr. \& Springer, vol. 56, no. 1, 2012, pp. 259-298

[3] D. H. Yu, Natural Boundary Integral Method and Its Application, Kluwer Academic Publishers, Beijing/New York, 2002.
[4] D. H. Yu, "Discretization of non-overlapping domain decomposition method for unbounded domains and its convergence", Mathematica Numerica Sinica, vol. 18, no. 4, pp. 2267-2272, 1996.

[5] D. H. Yu, J. M. Wu, "A Non-overlapping Domain Decomposition Method For Exterior 3-D Problem”, Comput. Math., vol. 19, no. 1, pp. 77-86, 2001.

[6] X. Q. Luo, Q. K. Du, L. B. Liu, "A D-N alternating algorithm for exterior 3-D Poisson problem with prolate spheroid boundary", Appl. Math. \& Comput., vol. 269(C), pp. 252-264, 2015.

[7] D. H. Yu, Z. P. Jia, "Non-overlapping domain decomposition algorithm based on natural boundary reduction for two dimensional Helmholtz equation", Mathematica Numerica Sinica, vol. 22, no. 2, pp. 228-240, 2000.

[8] Y. J. Chen, Q. K. Du, "Infinite concave angle area of anisotropic problem of non-overlapping domain decomposition algorithm", Mathematical applicate sinica, vol. 37, no. 5, pp. 912-925, 2014.

[9] Y. J. Chen, Q. K. Du, “A non-overlapping domain decomposition method for an anisotropic problem in an infinite domain with a concave angle”, Acta. Math. Appl. Sinica, vol. 37, no. 5, pp. 912-924, 2013. 